\title{
An Application-Level QoS Comparison of Single-Stream and Multi-Stream Approaches in a Wireless Ad Hoc Network
}

\author{
Toshiro Nunome and Shuji Tasaka \\ Department of Computer Science and Engineering, Graduate School of Engineering, \\ Nagoya Institute of Technology, Nagoya 466-8555, Japan \\ Tel: +81-52-735-7785, Fax: +81-52-744-3516 \\ e-mail: \{nunome, tasaka\}@nitech.ac.jp
}

\begin{abstract}
This paper compares application-level QoS of four schemes for live audio and video streaming in a wireless ad hoc network by simulation. There are two approaches to audio-video transmission: single-stream and multi-stream. The former transmits a single transport stream of interleaved audio and video, while the latter treats the two media as separate transport streams. Each approach has an alternative of whether media synchronization control at the destination is carried out or not. Thus, we have the four basic schemes. In the simulation, we also assess the network-level QoS. We then show that the multi-stream approach with media synchronization control is the best scheme among the four schemes.
\end{abstract}

\section{INTRODUCTION}

The rapid advancement in portable computing platforms and wireless communication technology has led to significant interest in wireless ad hoc networks [1]. They are networks with no fixed infrastructures, such as underground cabling or base stations, where all nodes are capable of moving and can be connected dynamically in an arbitrary manner. Each mobile host acts as a router, which discovers and maintains routes to other hosts and forwards packets for them in the network.

Some applications of ad hoc networks require the ability to support real-time multimedia streams such as live audio and video over the network. Then, the assessment of its quality is very important. The Internet Protocol (IP) suite plays an important role even in ad hoc networks. Then, we should assess the quality in IP-based ad hoc networks.

Owing to the layered architecture of IP-based networks, its Quality of Service $(Q o S)$ also has a layered structure. We can identify six levels of QoS: physical-level, link-level, networklevel, end-to-end-level, application-level and user-level [2]. For the users, the subjective quality (i.e., user-level QoS) is the most important QoS; it is closely related to application-level QoS. The preservation of the temporal structure is essential to high application-level QoS of continuous media [2].

When we transmit the continuous media streams in ad hoc networks, the temporal structure of the streams may be disturbed largely by delay and its jitter. In wireless networks such as IEEE 802.11, terminals share the same physical channel. Furthermore, wireless networks have slower transmission rates than wired ones. Thus, its delay easily increases, and its throughput largely decreases. In addition, the media access control (MAC) protocol usually has a carrier-sensing capability and a retransmission-based error recovery mechanism in order to recover transmission errors in the wireless channel. This also increases network delay and its jitter. Thus, in order to preserve the temporal relation, we need the media synchronization control [3], which is application-level QoS control.
We identify three types of media synchronization: intrastream synchronization, inter-stream synchronization and interdestination (or group) synchronization. The intra-stream synchronization control is necessary for the preservation of the timing relation between media units $(M U s)$ such as video frames in a single media stream; an MU is the information unit for media synchronization. The inter-stream synchronization is required for keeping the temporal relations among MUs in multiple media streams; synchronization between audio and video (i.e., lip$s y n c$ ) is a typical example. The inter-destination synchronization adjusts the output timing of each MU multicast to two or more destinations so that the MU can be output simultaneously at all the destinations.

A variety of studies on continuous media transmission in wireless ad hoc networks have been reported. However, most of them do not focus on the application-level or user-level QoS. In [4], $\mathrm{Hu}$ and Johnson describe the design and demonstration of a set of simple routing protocol mechanisms that substantially improve the transmission of real-time audio-video streams over an ad hoc network. They employ DSR (Dynamic Source Routing) [5] for the ad hoc routing and Microsoft Windows NetMeeting as the audio and video communication software. They use the measured signal-to-noise ratio (SNR) for received packets to detect when a route in use is likely to break soon. However, they show no quantitative result of the performance. On the other hand, in [6], Ruiz et al. propose an application-level QoS control scheme for multicast multimedia streaming. In order to achieve good user-level QoS, it changes codec, code-specific parameters and frame sizes of audio and video according to the network condition, which is measured with the percentage of packet loss. They apply the control to audio and video multicasting in an ad hoc network. They show the effectiveness in terms of the packet loss rate and the maximum delay jitter of received packets. However, they do not assess the temporal quality of continuous media.

In audio-video transmission, we treat two media streams at the same time. However, we scarcely find QoS studies handling both audio and video together at the application- and userlevels. There are two approaches to audio-video transmission: single-stream and multi-stream. The former transmits a single transport stream of interleaved audio and video. The international standards or recommendations activity of multimedia multiplexing methods often takes the single-stream approach. This includes ISO/IEC 13818-1 [7] for the Transport Stream and the Program Stream of MPEG 2. The latter treats the two media as separate transport streams.

Advantages and disadvantages of each approach have not been identified and evaluated sufficiently in wireless ad hoc networks. In [8], Tasaka et al. implement the four schemes, which are characterized by the number of transport streams treated (single or multi) and the existence or nonexistence of the media synchronization capability at the destination, on an infrastructure mode wireless LAN for stored MPEG video and voice. Furthermore, 
TABLE I

DEFINITION OF FOUR SCHEMES

\begin{tabular}{|l|c|c|}
\hline & single-stream & multi-stream \\
\hline \hline no control & type 0 & type 2 \\
\hline media synchronization control & type 1 & type 3 \\
\hline
\end{tabular}

in [9], Tasaka and Ishibashi employ live JPEG video and voice and compare application-level QoS of the four schemes in an integrated wired and wireless network. However, QoS assessment of the schemes in wireless ad hoc networks has not been done yet.

In this paper, we compare the application-level QoS of the four schemes for live audio and video streaming in a wireless ad hoc network by simulation. We also assess the network-level QoS, which is measured at the network layer. Ad hoc routing protocols are the network layer protocols, and then the networklevel QoS reflects characteristics of ad hoc networks. By assessing the application-level QoS and network-level QoS jointly, we show the best scheme in the wireless ad hoc network.

The rest of this paper is organized as follows. Section II explains the single-stream and multi-stream approaches. Section III describes principles of the media synchronization scheme. Section IV illustrates a methodology for the QoS assessment, including the network configuration, simulation method and QoS parameters. The simulation results are presented and discussed in Section V.

\section{Media Transmission Scheme}

We consider the transmission of a voice stream and the corresponding video stream from a source to a destination over a wireless ad hoc network. A video frame is defined as a video $\mathrm{MU}$, and a voice packet consisting of a constant number of voice samples as a voice MU. In this paper, we assume that the capturing and encoding delay of a voice MU is longer than that of a video MU. This is because the capture duration of a voice MU equals the inter-MU time, while that of a video MU is just a moment.

References [8] and [9] define the four basic types of media synchronization mechanisms as shown in Table I. Each type is characterized by two factors: the number of transport streams treated (single or multi) and the existence or nonexistence of the media synchronization capability at the destination.

In ad hoc networks, these schemes may affect subjective quality of media streams largely owing to frequent changes of network conditions such as delay and packet losses. In addition, the single-stream and multi-stream approaches are different in the transmission timings of voice and video MUs. Thus, QoS comparison of these schemes is a necessary step for the progress in ad hoc multimedia communications.

We describe the implementations of the schemes at the source node below.

\section{A. Single-Stream Approach}

The single-stream approach forms a single composite stream by interleaving voice and video MUs in the order of their timestamps and transmits it. The interleaving serves as an effective mechanism for inter-stream synchronization.

The implementation of the single-stream approach in this paper first sends a voice MU when the capturing of the voice MU and that of a video MU are started at the same time. This is because voice is more sensitive to intra-stream synchronization error than video. Thus, in order to minimize the temporal disturbance of the voice stream, we treat voice MUs prior to video ones. However, note that the destination does not distinguish media types of MUs until their output.

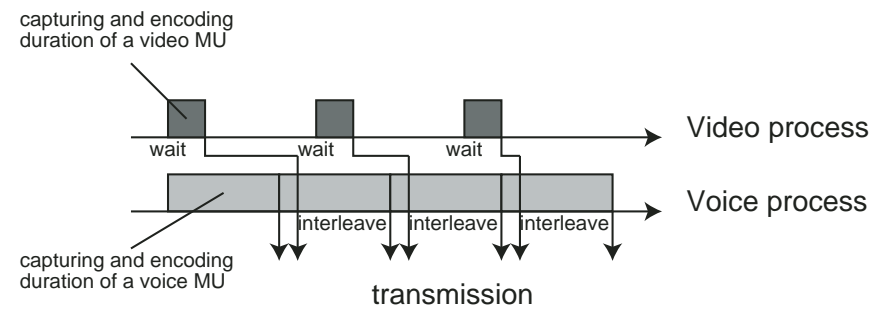

Fig. 1. Relations between capture and transmission timing in the single-stream approach.

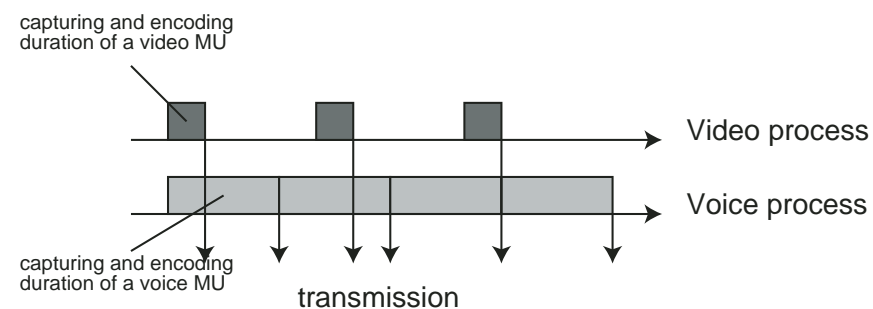

Fig. 2. Relations between capture and transmission timing in the multi-stream approach.

Figure 1 shows an example of relations between capture and transmission timing in the single-stream approach. In this approach, a video MU must wait until the end of capturing and encoding of voice MUs which started the capturing before the video MU. Then, the video MU is interleaved after the voice MUs.

\section{B. Multi-Stream Approach}

In the multi-stream approach, the voice and video are transmitted as two separate transport streams. Because each media stream is independent of the other, we can adopt network-level QoS control such as DiffServ [10] easily.

Figure 2 shows an example of relations between capture and transmission timing in the multi-stream approach. Regardless of the media type, this scheme tries to transmit captured MUs immediately.

Note that the two streams in the multi-stream approach are transmitted on the same route. This is because the routing is carried out by a pair of the source and destination addresses.

\section{Media Synchronization Algorithm}

In this paper, we employ the enhanced Virtual-Time Rendering (VTR) algorithm proposed in [11] for media synchronization control.

The original VTR algorithm [12] adaptively changes the buffering time according to the amount of delay jitter of MUs received at the destination. The enhanced VTR algorithm proposed in [11] also changes the buffering time according to MU loss for managing MU drop and retransmission. In this paper, we do not employ a retransmission-based error recovery at the application-level. However, the increased buffering time is used for error recovery mechanisms at the MAC layer.

The media synchronization control is effective in outputting MUs correctly when the out-of-sequence of MUs occurs. The out-of-sequence of MUs means that an MU arrives earlier than another MU which is transmitted by the source before the former. Whether the destination exerts the media synchronization control or not, the application layer of the destination receives the same number of MUs as the one received by the network layer in the same order. This is because media synchronization 


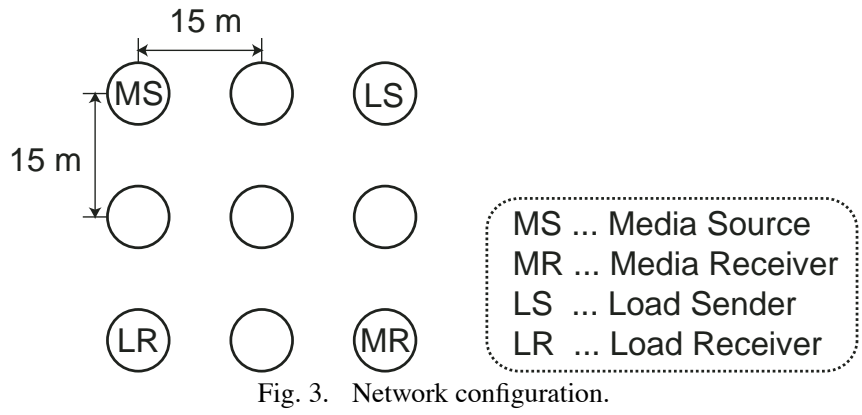

Fig. 3. Network configuration.

control is a kind of application-level QoS control, and only the destination carries out the control. However, when the destination exerts the media synchronization control, it buffers MUs. Then, it can correct the sequence of the MUs and can output them in the order of generation. On the other hand, when the destination does not carry out the media synchronization control, it just discards MUs received out of sequence.

Initially, in the enhanced VTR algorithm, the buffering time is set to a rough estimate of the maximum delay jitter, which is denoted by $J_{\max }[11]$; after the first MU is received, it can be changed by the modification of the target output time of each received MU. The target output time is the time when an MU should be output. When the MU arrives at the destination too late after the target output time, the target output time is delayed to absorb the jitter; this means increase in the buffering time. In order to preserve the real-time property of live media, we can set the maximum allowable delay $\Delta_{\text {al }}[11]$ so that the modification of the target output time does not make MU delay exceed this limit. Furthermore, the target output time can be advanced when the amount of delay jitter decreases; this means that the buffering time decreases. The algorithm uses the parameter $r$ for controlling the target output time.

The single-stream approach interleaves voice and video MUs into a composite stream. In the approach, the destination applies only intra-stream synchronization control to the composite stream. That is, the composite stream modifies the target output time.

In the multi-stream approach, the voice is selected as the master stream and the video as the slave stream since voice is more sensitive to intra-stream synchronization error than video. Only the master stream can modify the target output time for itself, and accordingly the slave stream modifies it by the same amount at the same time. Furthermore, in the multi-stream approach, we also employ inter-stream synchronization control.

\section{Methodology for Quality Assessment}

We compare the application-level QoS of the media transmission schemes by computer simulation with $n s-2$ [13].

\section{A. Network Configuration}

In this paper, we consider a grid topology network, which consists of 9 nodes as shown in Fig. 3. The interval between two vertical or horizontal adjacent nodes is constant, $15 \mathrm{~m}$.

We consider a detailed simulation model which is based on the distributed coordination function (DCF) [14] of the IEEE 802.11 wireless LAN. We employ the free space propagation model implemented in ns-2. Each node has an omni-directional antenna. The radio model uses system parameters similar to a commercial radio interface, Lucent Technologies' Orinoco 802.11b $11 \mathrm{Mbps}$ PC card; that is, we assume IEEE 802.11b. In the simulation, the transmission range of each node is about $22.49 \mathrm{~m}$. That is, the nodes are only within range of their immediate neighbors.
TABLE II

SPECIFICATIONS OF THE VOICE AND VIDEO.

\begin{tabular}{|l|c|c|}
\hline \multicolumn{1}{|c|}{ item } & voice & video \\
\hline \hline coding scheme & $\begin{array}{c}\text { ITU-T } \\
\text { G.711 } \mu \text {-law }\end{array}$ & $\begin{array}{c}\text { MPEG1 } \\
\text { GOP I }\end{array}$ \\
\hline image size [pixels] & - & $192 \times 144$ \\
\hline original average MU size [bytes] & 320 & 2000 \\
\hline original average MU rate [MU/s] & 25.0 & 20.0 \\
\hline original average inter-MU time [ms] & 40.0 & 50.0 \\
\hline original average bit rate [kbps] & 64.0 & 320.0 \\
\hline measurement time [s] & \multicolumn{2}{|c|}{120.0} \\
\hline
\end{tabular}

\section{B. Method of Simulation}

We assume MS (Media Source) as the voice and video sources. MS unicasts the media streams to MR (Media Receiver) with RTP/UDP. In the simulation, we employ DSR as the ad hoc routing protocol. DSR is a reactive routing protocol and operates entirely on demand. In the protocol, each node has no routing table. The source node decides an entire route to the destination and attaches the routing information to packets sent by the source. Nodes relay the packets according to the information.

We use a voice stream of ITU-T G.711 $\mu$-law and an MPEG1 video stream. Table II shows the specifications of the voice and video. Furthermore, we take the media capturing and encoding delay time into consideration in the simulation. The capture duration of a voice MU equals the inter-MU time, which is $40 \mathrm{~ms}$ in this paper, and the encoding time is negligible; therefore, we set the capturing and encoding delay time of each voice MU to $40 \mathrm{~ms}$. On the other hand, the capture duration of a video MU is just a moment. However, it spends much time to encode a video frame. In this paper, we set the capturing and encoding delay time of each video MU to $8 \mathrm{~ms}$, which is the same time as that in the experimental system in [11] ${ }^{1}$. Each MU leaves the source the capturing and encoding delay time after its timestamp. In addition, we assume that the capturing start time of the first voice MU and that of the first video MU are the same.

The parameter values in the enhanced VTR algorithm are set as follows. We set $r$ to $10 \mathrm{~ms}$ in order to prevent drastic changes of the target output time. The other thresholds and parameters in the VTR algorithm have the same values as those in [11]. That is, we set $J_{\max }$ and $\Delta_{\mathrm{al}}$ to $100 \mathrm{~ms}$ and $300 \mathrm{~ms}$, respectively. In the multi-stream approach, we exert loosely-coupled inter-stream synchronization control [12].

LS (Load Sender) and LR (Load Receiver) are used to handle traffic flows of interference. We also employ DSR for the load traffic. LS generates fixed-size IP datagrams of 1500 bytes each at exponentially distributed intervals and then transmits them to LR. The amount of the interference traffic is adjusted by changing the average of the interval. We refer to the average amount of the interference traffic as the average load.

\section{QoS Parameters}

We regard the quality of media synchronization as the major part of the application-level QoS in this paper. Thus, we need QoS parameters which reflect the media synchronization quality.

For the quality assessment of intra-stream synchronization for voice or video, we evaluate the coefficient of variation of output interval, which is defined as the ratio of the standard deviation of the MU output interval of a stream to its average and represents the smoothness of output of a media stream.

\footnotetext{
${ }^{1}$ In [11], JPEG is employed for video codec. On the other hand, this paper handles MPEG video. However, because of the GOP pattern in this paper, we have assumed that the capturing and encoding delay time of each MU is approximately the same as that of JPEG video in [11].
} 
We have also assessed inter-stream synchronization quality in the simulation. As a result, we noticed that all the schemes have high inter-stream synchronization quality. Thus, we do not show the result in this paper.

For the assessment of transfer efficiency, we use the $M U$ loss rate, which is the ratio of the number of MUs lost to the total number of MUs generated.

The average $M U$ delay, which is the average time of $M U$ de$l a y$, is a key measure for live media. The MU delay is defined as the time interval from the moment an MU is generated until the instant the MU is output.

Furthermore, we assess the number of route errors and the average number of hops as the network-level QoS parameters.

The number of route errors represents the number of route destruction between MS to MR during a simulation run. When a route in use breaks, the intermediate node returns a route error packet to the source.

As the number of hops from the source to the destination increases, the transfer delay also increases; it degrades the realtime property. In the simulation, the minimum number of hops from the source to the destination is two. However, if the route error occurs, the source searches an alternative route for transmission of packets. That is, the source may find a route with many hops.

\section{Simulation Results}

We first discuss the results of the network-level QoS assessment. Then, we show the application-level QoS of the four schemes.

Each symbol in the figures to be shown represents the average of 10 measured values which were obtained by changing the random seed for generating the interference traffic. We also show $95 \%$ confidence intervals of the QoS parameters in the figures. However, when the interval is smaller than the size of the corresponding symbol representing the simulation result, we do not show it in the figures.

\section{A. Network-Level QoS Assessment}

The number of route errors and the average number of hops, which are assessed at the network-level, are not affected by the media synchronization control. Thus, we show the results of types 1 and 3; both schemes exert the media synchronization control.

Figure 4 depicts the number of route errors which occurred with transmitted packets from the source during a simulation run versus the average load. In this figure, we find that for all the average loads here, the number of route errors with type 3 (i.e., the multi-stream approach) is smaller than or approximately the same as that with type 1 (i.e., the single-stream approach). This is due to bursty transmission from the source in the single-stream approach. The bursty traffic cause much collision at the MAC layer. If a packet which contains a part of a voice or video MU is dropped owing to the collision with a packet of the load traffic, the intermediate node generates a route error packet. Thus, as the traffic becomes bursty, the number of route errors increases.

Next, we discuss the routing strategy of DSR for transmitted stream from the source. In the network topology, the minimum number of hops from the source to the destination is two; this route is shown in Fig. 5(a). However, if a packet which contains a part of a voice or video MU is dropped owing to the collision with a packet of the load traffic, DSR detects route destruction and then searches an alternative route. Figure 6 depicts the average number of hops for the multi-stream approach as a function of time. We measured the average every second. This figure shows the results when the average loads are $500 \mathrm{kbps}$ and 1.2 Mbps. We see in the figure that the fluctuation of the average

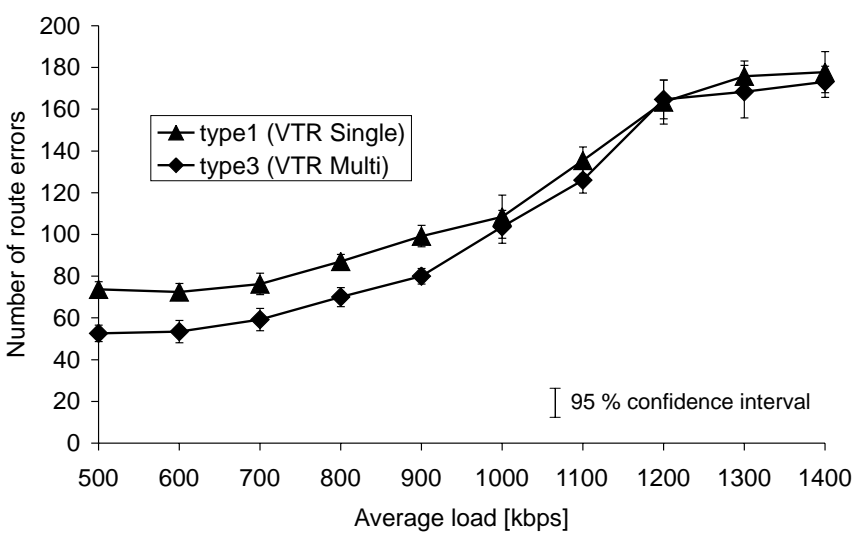

Fig. 4. Number of route errors.

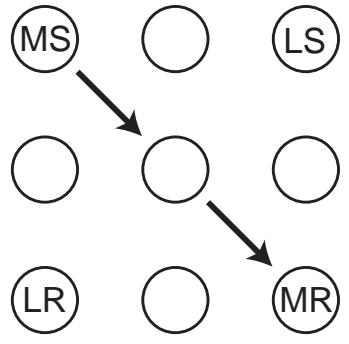

(a) Minimum number of hops.

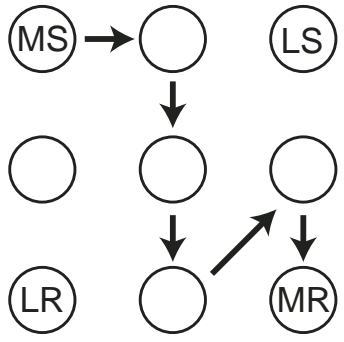

(b) Example of maximum number of hops.
Fig. 5. Selected routes in the simulation.

number of hops under the 1.2 Mbps load condition is larger than that under the $500 \mathrm{kbps}$ condition. This is because the amount of route errors increases as the average load increases; we have noticed this in Fig. 4. In the simulation, we have observed that the maximum number of hops is five. We show an example of those routes in Fig. 5(b). Since the number of hops varies during each simulation run, network delay fluctuates largely.

\section{B. Application-Level QoS Assessment}

1) Transfer Efficiency: Figure 7 displays the MU loss rate of video versus the average load.

In Fig. 7, we notice that for the average loads larger than $1.0 \mathrm{Mbps}$, types 1 and 3 has lower MU loss rates of video than

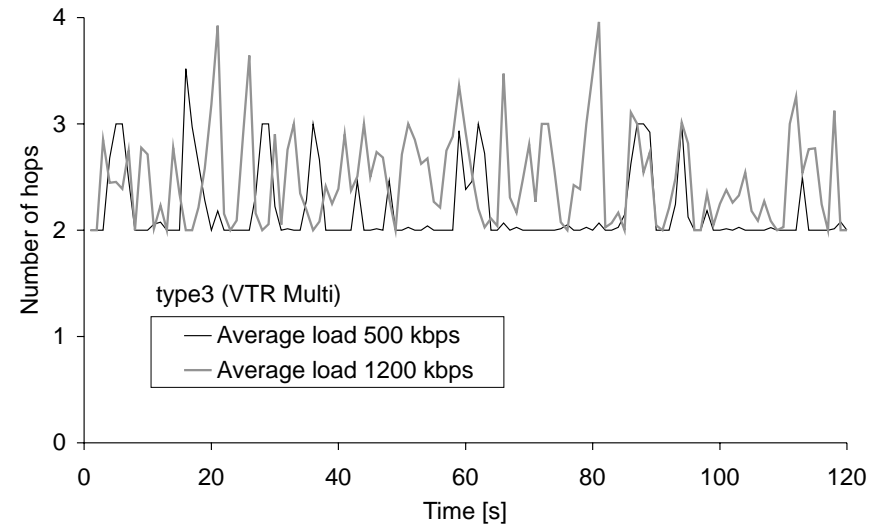

Fig. 6. Average number of hops in multi-stream approach versus time. 


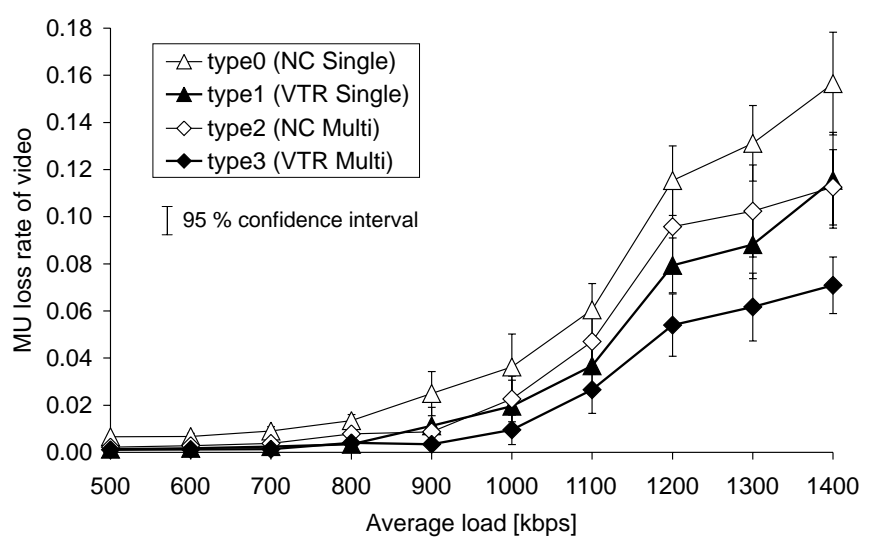

Fig. 7. MU loss rate of video.

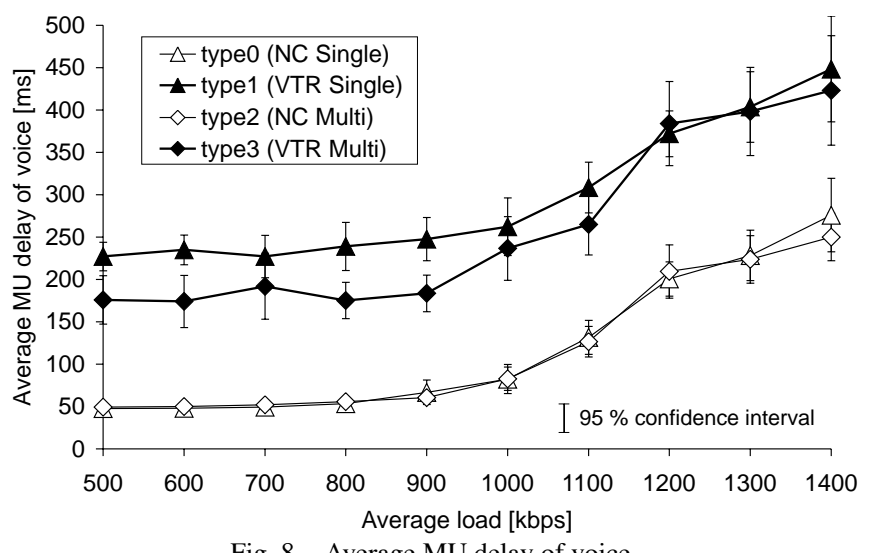

Fig. 8. Average MU delay of voice.

the other types; types 1 and 3 exert the media synchronization control. This is because the sequence of MUs arrived at the destination may be disturbed by route changes. When the destination exerts the media synchronization control, it buffers MUs. Then, it can correct the sequence of the MUs and can output them in the order of generation.

We see in Fig. 7 that for all the average loads here, the MU loss rate of video with type 0 is higher than that with type 2 . Furthermore, we find that when the average load is larger than about $900 \mathrm{kbps}$, the MU loss rate of video with type 1 is higher than that with type 3 . That is, we can say that the single-stream approach has dropped more video MUs than the multi-stream approach. This is because the traffic transmitted from the source in the single-stream approach is more bursty than that in the multi-stream approach. Thus, the number of dropped packets increases in the single-stream approach. Furthermore, in the single-stream approach, a video MU is interleaved after the voice MUs which started the capturing before the video MU; that is, video MUs are affected by voice MUs transmission. Therefore, the video MU loss increases in the single-stream approach.

2) Real-Time Property: Figure 8 displays the average MU delay of voice.

We observe in Fig. 8 that the average MU delays with the schemes which exert the media synchronization control (i.e., types 1 and 3) are larger than that with the other schemes. This is because the media synchronization control buffers MUs in order to absorb network delay jitter. We also find in this figure that for the average loads lighter than about $1.1 \mathrm{Mbps}$, the average MU delay for type 1 , which employs the single-stream approach, is larger than that for type 3 . This is because the single-stream approach exerts intra-stream synchronization control without dis-

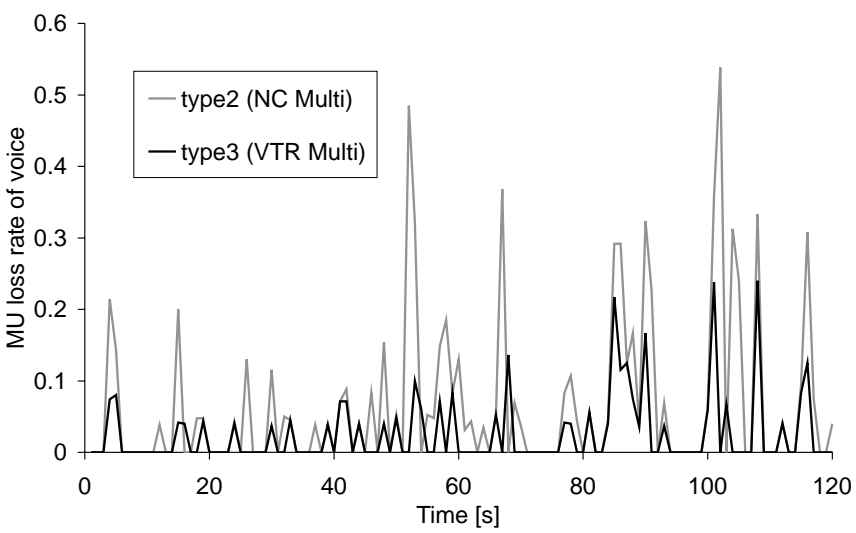

Fig. 9. MU loss rate of voice versus time (Average load $=1200 \mathrm{kbps}$ ).

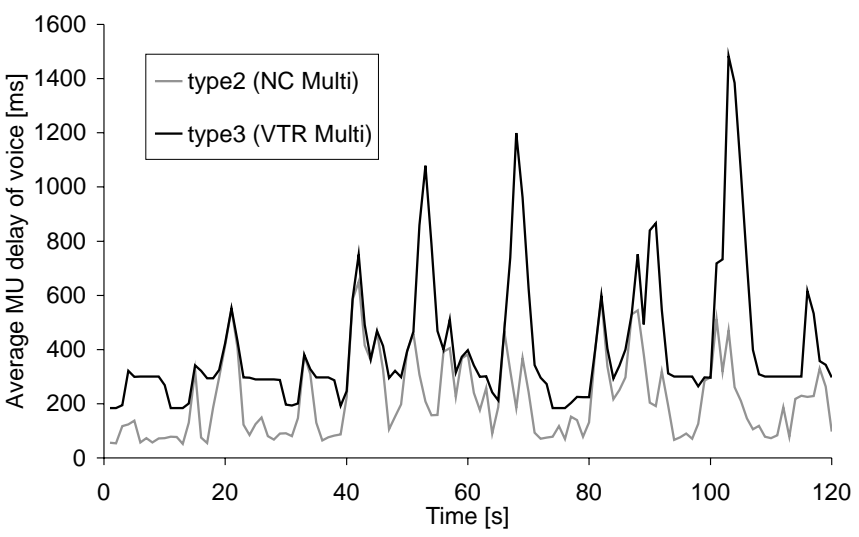

Fig. 10. Average MU delay of voice versus time (Average load = $1200 \mathrm{kbps}$ ).

tinction of the media types. In the multi-stream approach, only the master stream (i.e., voice stream) can modify the target output time for itself. On the other hand, the arrival time of video MUs, which have larger delay than voice ones, is also used to control the target output time in the single-stream approach. Therefore, when the media synchronization control is exerted, the single-stream approach causes larger MU delay than the multi-stream approach.

3) Tradeoff between Transfer Efficiency and Real-Time Property: Here, we discuss the relationship between the transfer efficiency and real-time property in the multi-stream approach. For this purpose, we show the MU loss rate of voice as a function of time in Fig. 9. Figure 10 depicts the average MU delay of voice versus time. In these figures, we measured the quality every second; the measurement was made for 120 seconds after the capturing of the first MU. We find in the figures that both the MU loss rate and the average MU delay fluctuate largely. This is due to frequent route changes as shown in Fig. 6.

In Fig. 9, we observe that types 2 and 3 incur the MU loss at approximately the same time. However, the number of MU loss with type 3 is smaller than that with type 2 . On the other hand, we see in Fig. 10 that these types also cause large MU delay at approximately the same time; the amount of MU delay for type 2 is smaller than that for type 3 . In addition, the time when the MU loss rate becomes high in Fig. 9 almost agrees with the time when the MU delay shows a sharp peak in Fig. 10. That is, the relationship between the types in the MU loss rate is opposite to that in the average MU delay, and the spikes of the MU delay correlate with those of the MU loss rate. The reason is as follows. In type 3, when the arrival of an MU largely delays, the target output time is delayed by the media synchronization con- 


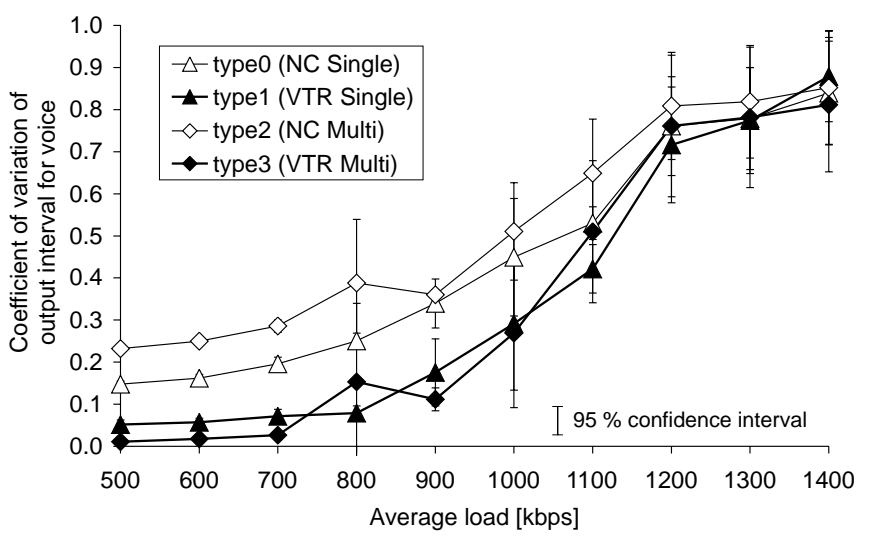

Fig. 11. Coefficient of variation of output interval for voice.

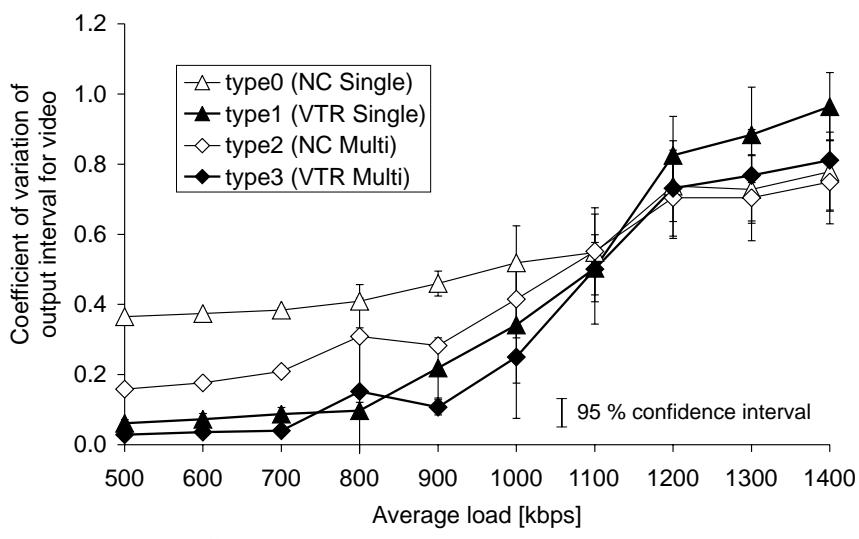

Fig. 12. Coefficient of variation of output interval for video.

trol; this means increase in the buffering time. However, owing to the large buffering time, the destination can output much MUs received out of sequence.

4) Media Synchronization Quality: Figure 11 depicts the coefficient of variation of output interval for voice as a function of the average load. Figure 12 plots the coefficient for video versus the average load.

We find in Figs. 11 and 12 that for the average loads smaller than around 1.1 Mbps, types 1 and 3 have smaller coefficients of variation of output interval than types 0 or 2 . On the other hand, in Fig. 12, when the average load is larger than about $1.2 \mathrm{Mbps}$, the coefficient for video with type 1 and that with type 3 are larger than that with the others. This is because the parameter settings of the enhanced VTR algorithm may not be appropriate on that load condition; the optimization of the parameters is a future study.

Furthermore, we have investigated the subjective quality by playing the media according to the simulation results. As a result, we found that types 1 and 3 have better quality than types 0 and 2. However, when we felt large pauses, which appear as spikes in Fig. 10, the output quality deteriorated in every type. In addition, we felt that the output voice quality of type 0 is the worst among the four schemes. However, we did not perceive the difference in video quality among the schemes.

\section{CONCLUSIONS}

In this paper, we have compared the application-level QoS of four schemes for live audio and video streaming in a wireless ad hoc network by simulation. As a result, we found that the media synchronization control can reduce the MU loss. This is because the sequence of MUs arrived at the destination is disturbed by route changes; this is the main difference from the results in [8] and [9]. In addition, we found that the singlestream approach has dropped more video MUs than the multistream approach. This is because the traffic transmitted from the source in the single-stream approach is more bursty than that in the multi-stream approach. Thus, more video MUs drop in the single-stream approach. From the above discussion, we can say that the multi-stream approach with the media synchronization control achieves high quality of media synchronization.

In this paper, we assumed unicast communications and employed DSR for the ad hoc routing protocol; the routing was carried out by a pair of the source and destination addresses. Thus, in the multi-stream approach, the two streams were transmitted on the same route. On the other hand, in multicast communications, each stream may have a different multicast address and a different route; this case is a subject for future study. Furthermore, we should assess the QoS in other network configurations which are representative of the real world, such as mobile nodes and varying node distances.

\section{ACKNOWLEDGMENT}

This work was supported by the Grant-In-Aid for Young Scientist (B) of Japan Society for the Promotion of Science under Grant 15760262 and the Hori Information Science Promotion Foundation.

\section{REFERENCES}

[1] Mobile ad-hoc networks (MANET) charter, (http://www.ietf.org/html.charters/manet-charter.html).

[2] S. Tasaka and Y. Ishibashi, "Mutually compensatory property of multimedia QoS," in Conf. Rec. IEEE ICC 2002, pp.1105-1111, Apr./May 2002.

[3] G. Blakowski and R. Steinmetz, "A media synchronization survey: Reference model specification, and case studies," IEEE J. Sel. Areas in Commun., vol. 14, no. 1, pp. 5-35, Jan. 1996.

[4] Y-C. Hu and D. B. Johnson, "Design and demonstration of live audio and video over multihop wireless ad hoc networks," Proc. MILCOM 2002, pp.1211-1216, Oct. 2002.

[5] D. B. Johnson, D .A. Maltz and Y-C. Hu, "The dynamic source routing protocol for mobile ad hoc networks (DSR)," Internet Draft, draft-ietf-manet-dsr-09, Apr. 2003.

[6] P. M. Ruiz, A. F. Gomez-Skarmeta, P. Martinez, J. A. Sanchez and E. Garcia, "Effective multimedia and multi-party communications on multicast MANET extensions to IP access networks," in Proc. International Conference on Information Networking (ICOIN) 2003, Feb. 2003.

[7] Information technology - Generic coding of moving pictures and associated audio information: Systems, ISO/IEC 13818-1, ITU-T Recommendation H.222.0, (Nov. 1994).

[8] S. Tasaka, H. Nakanishi and Y. Ishibashi, "Dynamic resolution control and media synchronization of MPEG in wireless LANs," Conf. Rec. IEEE GLOBECOM'97, pp. 138-144, Nov. 1997.

[9] S. Tasaka and Y. Ishibashi, "A performance comparison of single-stream and multi-stream approaches to live media synchronization,' IEICE Trans. Commun., vol.E81-B, no.11, pp. 1988-1997, Nov. 1998.

[10] S. Blake, D. Blake, M. Carlson, E. Davies, Z. Wang and W. Weiss, "An architecture for differentiated services," RFC 2475, Dec. 1998.

[11] S. Tasaka, T. Nunome and Y. Ishibashi, "Live media synchronization quality of a retransmission-based error recovery scheme," Conf. Rec. IEEE ICC 2000, pp. 1535-1541, June 2000.

[12] Y. Ishibashi and S. Tasaka, "A synchronization mechanism for continuous media in multimedia communications," in Proc. IEEE INFOCOM'95, pp. 1010-1019, Apr. 1995.

[13] The network simulator - ns-2 -, (http://www.isi.edu/nsnam/ns/)

[14] Wireless LAN Medium Access Control (MAC) and Physical Layer (PHY) Specifications, ISO/IEC 8802-11: 1999(E), ANSI/IEEE Std 802.11 1999 edition, (Aug. 1999). 